\title{
Contribuciones de la formación en el área de materiales
}

\author{
| Juan Manuel Vélez Restrepo**
Juan Manuel Jaramillo***
}

Recibido: 19/05/2014 - Aceptado: 27/06/2014

\begin{abstract}
Resumen
Este artículo de reflexión sustenta que el conocimiento sobre materiales y su procesamiento son factores clave del desarrollo tecnológico e industrial. Se muestra la relación entre la dinámica de la innovación y la tecnología de materiales, evidenciada en las revoluciones industriales de los últimos siglos. Igualmente se plantea el reto que enfrenta el área del conocimiento en materiales ante la escasez de materia prima y la disminución del impacto ambiental derivado de la transformación y la deposición final. Esto ha generado la necesidad de fortalecer la formación científica y profesional en materiales para respaldar los sistemas productivos nacionales y generar valor agregado. Se presenta como evidencia la importancia estratégica de los materiales en diferentes países para el desarrollo tecnológico. Finalmente el fuerte incremento de programas de postgrado en el área de materiales en Colombia se discute frente al reducido número de programas de pregrado en Ingeniería de materiales.
\end{abstract}

Palabras clave: ingeniería, materiales, tecnología, competitividad, industria, innovación.

\footnotetext{
* Artículo de reflexión no derivado de investigación

** Profesor de la Universidad Nacional de Colombia Sede Medellín. Doctor en materiales. E-mail: jmvelez@unal. edu.co. Dirección: Carrera 80 N. ${ }^{\circ}$ 65-223 - Núcleo Robledo. Teléfono: 4255000. Fax: 4309311

*** Profesor de la Universidad EAFIT. Doctor en Ing. Eléctrica. E-mail: jjaram44@eafit.edu.co. Dirección: Carrera 49 N. ${ }^{\circ} 7$ Sur - 50. Teléfono: 2619500. Fax: 2619381
} 


\title{
Contributions in formation in materials science and engineering
}

\begin{abstract}
This article sustains that knowledge of materials and their processing are key factors of technological and industrial development. The industrial revolutions of the past centuries are evidence of the relationship between the dynamics of innovation and technology of materials. The materials knowledge field has as principal challenges confront the scarcity of raw materials and the reduction of environmental impact due to processing and final waste disposal. This has generated the need to strengthen the scientific and professional training materials to support national production systems and create value. The materials is a strategic area in many developed countries due to its importance of materials on the technology development. Finally, the article discuss the sharp increase in graduate programs in the area of materials against the small number of undergraduate programs in engineering materials.
\end{abstract}

Key Words: Materials, Engineering, Technology, competitiveness, Innovation, Industry. 


\section{INTRODUCCIÓN}

La ciencia, la tecnología, la ingeniería y la innovación juegan un papel fundamental en el crecimiento económico y la mejora de la calidad de vida de los ciudadanos. Estos son los motores del desarrollo integral. Generan empleo y bienestar a través de la innovación y la comercialización de nuevos productos y servicios, y son esenciales para la construcción de nuevas capacidades tecnológicas que son básicas para las naciones de las Américas en el siglo XXI [1]. El desarrollo de nuevas tecnologías ha estado asociado con el desarrollo de los materiales adecuados para las aplicaciones específicas. El avance en el conocimiento de un tipo de material puede ser una base importante para la progresión paso a paso de una tecnología. Como ejemplos se puede citar el impacto de la disponibilidad de acero de bajo costo en la industria del automóvil, y de los materiales semiconductores en la industria de los dispositivos electrónicos utilizados en la era contemporánea [1-3].

El conocimiento sobre los materiales y su procesamiento constituyen un factor clave para la capacidad tecnológica de las empresas o sectores, y definen su competitividad en el mercado global. Según la OEA, la investigación en materiales y la nanotecnología son importantes para el desarrollo económico, ofrecen enormes oportunidades para las economías de las Américas y contribuyen al beneficio de sus sociedades [1]. Además, el sector económico y tecnológico de materiales, que incluye el procesamiento de materias primas, la producción de semielaborados, el diseño de nuevos materiales y procesos para aplicaciones específicas, el comercio de insumos y el reciclaje, es considerado estratégico por varias naciones desarrolladas y organismos multilaterales.

Los profesionales y científicos de la ciencia y de la ingeniería de materiales trabajan en el desarrollo de los materiales necesarios para los nuevos productos, diseñan mejores rutas de fabricación, y a menor costo, e impulsan el mejoramiento del desempeño de los materiales existentes. En su trabajo debe estar presente el impacto ambiental y la sostenibilidad de sus productos, incluyendo criterios de ciclo de vida de los materiales. Otro campo de acción es la optimización de la selección de materiales y la creación y promoción del uso de bases de datos sofisticadas a partir de las cuales se pueden predecir las propiedades y el comportamiento en servicio de materiales y componentes.

El presente artículo hace una corta exposición de la dinámica de la innovación y su relación con la tecnología de materiales. También se presenta la evolución del área del conocimiento en ciencia e ingeniería de materiales en el escenario global, así como los retos y oportunidades que enfrenta de acuerdo los lineamientos de organismos multilaterales $[1,4]$. Se finaliza con la discusión sobre la necesidad de fortalecer los 
programas de ingeniería de materiales, y sus afines, como estrategia para ganar terreno en la competitividad internacional de productos y para el aumento del valor agregado a las materias primas producidas por el país.

\section{DINÁMICA DE LA INNOVACIÓN Y LA TECNOLOGÍA DE MATERIALES}

Los desarrollos tecnológicos generados por las revoluciones científicas e industriales han coexistido con los avances en el conocimiento de materiales. Durante la primera Revolución Industrial el desarrollo del ferrocarril, la implementación de la energía eléctrica, el motor de combustión interna, entre otras, demandaron una rápida tecnificación de la fabricación del acero y la búsqueda de otras aleaciones. La segunda revolución consolidó la industria química, eléctrica, de telecomunicaciones; la producción en masa y los sistemas de distribución aparecieron en escena; los talleres de enseñanza dieron paso a los laboratorios de investigación y desarrollo, tanto en universidades como en grandes corporaciones. Ya en la tercera revolución, llamada revolución científica y tecnológica, aparecen la robótica, la bioingeniería y biotecnología, y las tecnologías de información y comunicaciones. Las transformaciones tecnológicas y socioeconómicas ocurridas en cada uno de esos momentos históricos han generado la necesidad de nuevos materiales y procesos. En la actualidad los retos son mayores, dada la complejidad de los problemas a resolver para alcanzar índices aceptables de sostenibilidad económica y ambiental. Las bases científicas de la materia condensada y de la física de materiales han servido de apoyo a grandes descubrimientos que han impulsado tecnologías de vanguardia en diversos sectores de la economía, como se ilustra en la figura 1 .

Los nuevos materiales y la capacidad de transformación han posibilitado el desarrollo de computadores, equipos de comunicación, instrumentos científicos, aviones, carros, robots, entre otros. Esto ha sido el resultado del esfuerzo conjunto de los sistemas científico, tecnológico e industrial, y constituye una fuerza motriz para la innovación y el desarrollo económico y social; las innovaciones basadas en el conocimiento son la base para la competitividad de empresas, sectores y países en el escenario internacional [5, 6]. Se considera que cerca del $70 \%$ de las innovaciones tecnológicas se apoyan directa o indirectamente en las propiedades de los materiales que usan [7]. Las innovaciones en materiales pueden ser empleadas en sectores tecnológicos y ramas de la industria que tienen potencial aplicación para reducir la polución ambiental, economizar energía, preservar recursos, reducir el impacto negativo del transporte, entre otras.

Esta prospectiva ha sido aprovechada por los países cuyas economías se soportan en sectores de alta tecnología. Así, en Estados Unidos, The National Research Council reconoció la Ciencia e Ingeniería de materiales como un área estratégica, definida con una visión integral que relaciona los tres factores determinantes del desempeño del 
material o componente: composición, propiedades y procesos [8]. El Departamento de Comercio e Industria del Reino Unido (UK) estableció el área de materiales como una tecnología de apoyo para el estímulo de la innovación y el aumento de la productividad de la industria, basado en la importancia del sector en la economía: $15 \%$ del PIB y cerca de 1,5 millones de empleos directos [9]. La Academia Alemana de Ciencias e Ingeniería considera que los materiales son de gran importancia para la industria, ya que representan un mercado de un billón de euros y emplean alrededor de cinco millones de personas. Sin embargo, reporta falencias en la cadena material-producto final que dificultan la creación de productos innovadores basados en resultados de investigación, por lo que se recomendó estimular la transferencia de conocimiento, con énfasis en el intercambio de investigadores entre la industria y las instituciones de investigación científica [8]. En Taiwán, en 1980 el Gobierno definió cuatro tecnologías básicas para el desarrollo del país: materiales, electrónica, informática y la bioingeniería. La industria electrónica taiwanesa ha demandado mayor cantidad de recurso humano en el área de materiales con respecto al proyectado inicialmente [10], lo que generó el surgimiento de departamentos y programas curriculares de ciencia e ingeniería de materiales en el país. Finalmente, la Comunidad Europea creó en 1987 la Federation of European Materials Societies, formada por organizaciones o institutos de Inglaterra, Alemania y Francia y que hoy cuenta con 28 socios; la actividad que más identifica esa asociación es la realización del evento EuroMat, "European Congress and Exhibition on Advanced Materials and Processes".

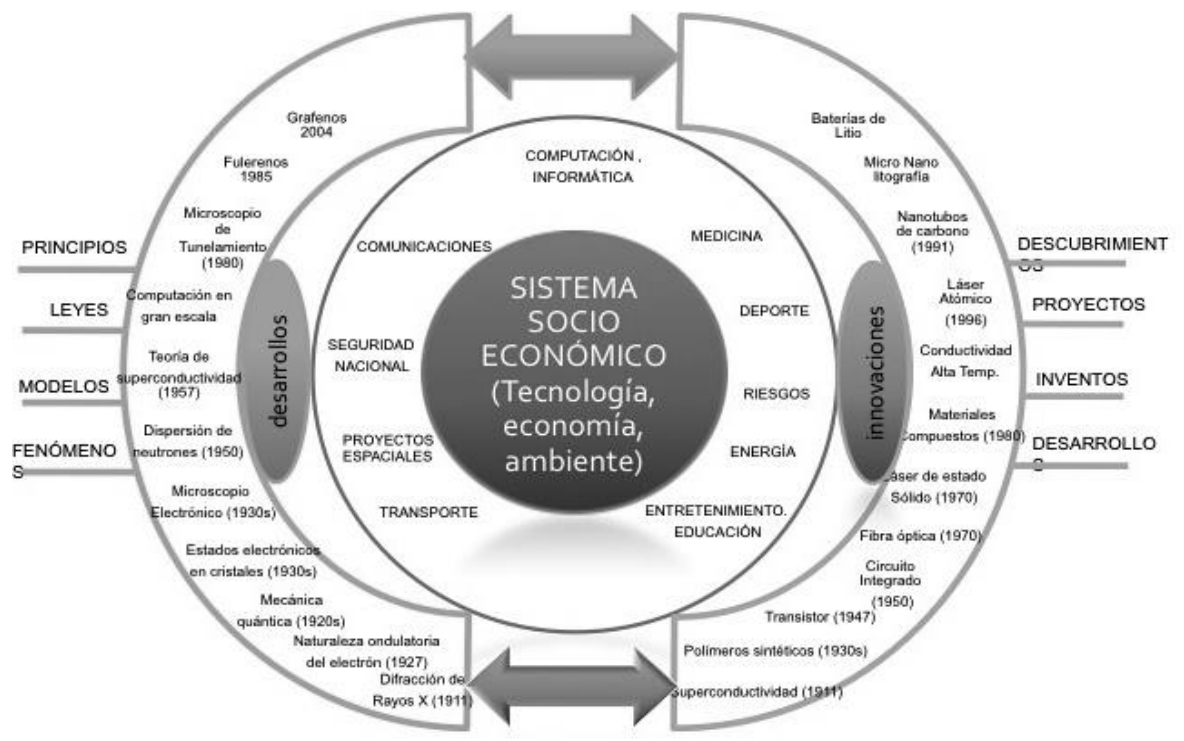

Figura 1. Impactos de los desarrollos científicos e innovaciones tecnológicas en el área de materiales en el desarrollo social. 


\section{RETOS Y OPORTUNIDADES DE LA CIENCIA Y LA INGENIERÍA DE MATERIALES}

El acelerado crecimiento de la población, la emisión de gases y la amenaza de escasez de recursos naturales emiten un mensaje de urgencia sobre la viabilidad del modelo socioeconómico basado en el consumo. Es necesario "desacoplar" el crecimiento económico de la extracción de recursos naturales y disminuir la intensidad del consumo de materiales [11]. Mantener los niveles de calidad de vida logrados en los países avanzados al tiempo que se permite el progreso económico y social de los países menos desarrollados y se minimiza el consumo de los recursos y el deterioro del ambiente es un reto complejo [11-14]. El desarrollo sostenible tiene como metas el bienestar humano, la viabilidad económica y la preservación del medio ambiente; eso implica reducir el consumo global de recursos y minimizar el impacto ambiental negativo. Para responder a este reto es necesario transformar el ciclo de producción material-producto final, o sea rediseñar los procesos y productos industriales, así como las formas de suministrar, empacar, transportar, etc. En conclusión, las políticas implementadas deberán traer como resultado concreto mayor eficiencia energética y reducción de la intensidad de consumo de materiales $[15,16]$. En este objetivo social los especialistas en materiales tienen roles importantes: diseñar nuevos materiales, mejorar la eficiencia en las aplicaciones, optimizar los procesos de producción y desarrollar tecnologías de reciclaje.

La tabla 1 presenta de forma resumida los retos tecnológicos del futuro en los cuales la ciencia y la ingeniería de materiales tienen oportunidades de participación en el desarrollo de nuevos productos, sistemas o materiales.

Tabla 1. Retos tecnológicos estratégicos para la ingeniería de materiales

Energía: Obtención de energía a partir de fuentes diferentes al carbono, "energías de bajo carbono"[17].
Solar: La mayor limitación en la energía solar es la baja eficiencia de conversión de las celdas solares, que oscila entre $10 \%$ y $20 \%$. Se requiere de sistemas para el almacenamiento de la energía colectada.

Eólica: Su costo hoy es un $25 \%$ del costo en 1980, mejora ganada con el aumento de tamaño y eficiencia de las turbinas. Los materiales deben mejorar la relación resistencia-densidad en los alabes; materiales que reemplacen las tierras raras en la fabricación de magnetos; mejorar la durabilidad de los generadores, ya que trabajan en ambientes agresivos (recubrimientos para protección frente a la erosión y el desgaste) [18].

Nuevos materiales, con alta relación resistencia/peso que permitan construcciones con menor consumo de materia prima; los métodos de fabricación átomo/átomo, molécula/molécula (nanotecnología) permitirán obtener materiales de altas especificaciones. Los materiales compuestos, con base en fibras naturales hacen posible la creación de estructuras ligeras, con buen desempeño, más amigables con el medio ambiente

Los procesos constructivos y sus métodos son un reto mayor; la eterna construcción manual hace ineficiente y costosa la construcción [4]. 


\begin{tabular}{|c|c|}
\hline $\begin{array}{l}\text { Infraestructura Urbana: } \\
\text { Se requiere de edificios más } \\
\text { eficientes desde el punto de } \\
\text { vista estructural, energé- } \\
\text { tico, de espacios, etc. [19] }\end{array}$ & $\begin{array}{l}\text { Iluminación: la evolución reciente ha sido vertiginosa, la eficiencia pasó del } \\
10 \% \text { en lámparas incandescentes al 95\% en lámparas LED; logros seme- } \\
\text { jantes se relatan en la durabilidad de los componentes. Pero esta tecnología } \\
\text { trae problemas ambientales asociados al desecho de las lámparas usadas: } \\
\text { la presencia de elementos tóxicos en el sistema demanda el desarrollo de } \\
\text { métodos de reciclaje y deposición final, nuevo reto para la ingeniería. }\end{array}$ \\
\hline $\begin{array}{l}\text { Transporte: el transporte } \\
\text { individual urbano es insos- } \\
\text { tenible [20]. }\end{array}$ & $\begin{array}{l}\text { Nuevas fuentes de potencia: el desarrollo del carro eléctrico es un éxito } \\
\text { en la reducción de la emisión de gases tipo invernadero pero genera retos } \\
\text { múltiples para el área de materiales: más eficiencia en la utilización de } \\
\text { materiales conductores (cobre), desarrollo de acumuladores de energía } \\
\text { (baterías), sistemas electrónicos, entre otros. } \\
\text { Vehículos más livianos: La relación potencia/peso interviene en la eficien- } \\
\text { cia total. El desarrollo de materiales más livianos reducirá el consumo de } \\
\text { combustible y la contaminación asociada a él. }\end{array}$ \\
\hline $\begin{array}{l}\text { Medio ambiente y mate- } \\
\text { rias primas [12] }\end{array}$ & $\begin{array}{l}\text { El escenario futuro prevé la masificación de las "tecnologías limpias" e } \\
\text { incrementará la demanda de materiales especiales (tierras raras), de poca } \\
\text { abundancia en la corteza terrestre. La amenaza de escases es un reto para } \\
\text { el sector de extracción y procesamiento de estos minerales y es un riesgo } \\
\text { para el sector tecnológico que utiliza esas materias primas. Se trata de una } \\
\text { oportunidad de nuevos desarrollos y nuevos negocios, que un país minero } \\
\text { como Colombia debería aprovechar. }\end{array}$ \\
\hline
\end{tabular}

Fuente: elaboración propia

\section{EL ÁREA DEL CONOCIMIENTO. RECURSO HUMANO EN CIENCIA E INGENIERÍA DE MATERIALES}

Estados Unidos inició en 1960 un programa nacional de creación de laboratorios interdisciplinarios (IDL). La estrategia reorientó la investigación en materiales de cara a la carrera aeroespacial, hasta entonces asociada a departamentos curriculares como química, física, mecánica, minas, metalurgia y civil, entre otros. Esta estrategia produjo impactos importantes: 1) la participación de especialistas de diferentes áreas permitió el desarrollo de productos y servicios con valor comercial, a partir de resultados científicos y tecnológicos; 2) la disponibilidad de infraestructura de investigación en diversas áreas del conocimiento; 3) una nueva estructura organizacional para la investigación que respondió a una iniciativa nacional y 4) una nueva modalidad de financiación de la investigación que produjo cambios en la gestión de las universidades. En resumen, surgió un nuevo contrato para la ciencia, que favoreció al investigador, a la institución $\mathrm{y}$ al ambiente en el que se mueve el estudiante de posgrado [21-23].

Durante algunas décadas se enfatizó la diferencia existente entre ingeniería de materiales y ciencia de materiales con una connotación tecnológica, a la primera, y cien- 
tífica, a la segunda. La ciencia de materiales investiga la relación entre la naturaleza de los materiales (composición, estructura) y sus propiedades. La ingeniería de materiales tiene como objetivo diseñar o seleccionar materiales y procesos para cumplir funciones específicas, con base en la relación estructura propiedades. Este último aspecto incluye la evaluación del desempeño del material cualitativa y cuantitativamente, de acuerdo con normas técnicas [24]. El trabajo conjunto de científicos e ingenieros ha producido grandes avances tecnológicos y ha impulsado la visión integral de la ciencia y la ingeniería de materiales que relaciona las propiedades, la composición y estructura, desde la escala nano y micro, y el proceso de obtención, factores determinantes en el desempeño del material, entendido como el cumplimiento de las funciones para las que fue diseñado dentro de parámetros técnicos, económicos y sociales. Esta visión se representa por el tetraedro mostrado en la figura 2, y se considera como una herramienta de gran importancia en la selección y diseño de materiales [9].

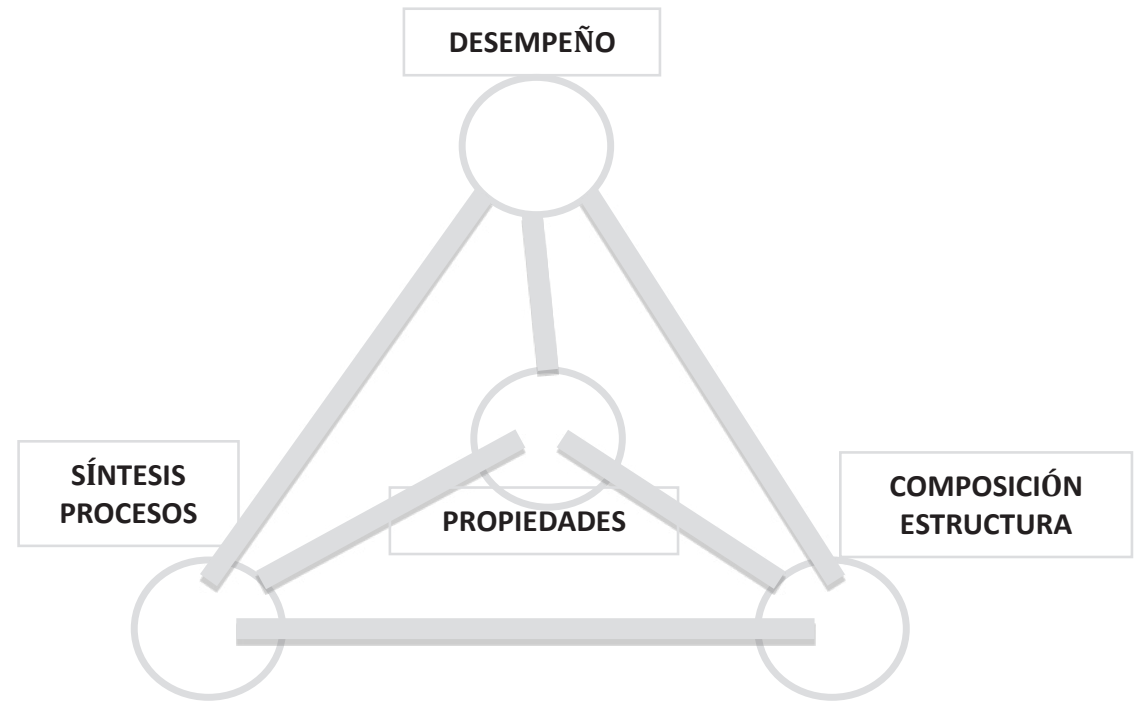

Figura 2. Tetraedro que representa los diferentes elementos de la ingeniería y ciencia de materiales.

Adaptación de los autores, con base en referencia [9]

En países desarrollados como Estados Unidos, Inglaterra, Alemania, Europa y Taiwán, el número de departamentos académicos y de programas curriculares en ciencia e ingeniería de materiales ha aumentado notablemente en las últimas décadas, resultado de esa visión interdisciplinaria [25-27]. En América Latina se observa gran actividad académica en el área de ingeniería de materiales. Brasil cuenta con 53 programas curriculares de pregrado activos en el área [28] y 46 programas de posgrado [29]. En 
Argentina se registran cinco programas de ingeniería de materiales y 12 programas de posgrado en ciencias o ingeniería de materiales [30]. En Chile se reportan 34 programas de pregrado en ingeniería civil, con énfasis en metalurgia, materiales, minerales o metalurgia extractiva, una actividad intensa de formación de recurso humano en el área para el tamaño del país [31]. En esos países se ha reconocido el papel estratégico de los materiales en el diseño de nuevos productos y maquinaria de buena calidad, a costos competitivos en el mercado global, teniendo en cuenta el mejoramiento continuo de los materiales existentes y el diseño de nuevos materiales e impulsando procesos tecnológicos que garanticen la protección del medio ambiente a pesar del aumento en el consumo de bienes y energía a escala global [32].

La obtención de productos tecnológicos o de consumo masivo es el resultado de la transformación de materias primas. En este ciclo de vida intervienen actividades primarias, que corresponden a la producción industrial que termina con el ensamblaje del producto final, y actividades de apoyo relacionadas con capital intangible y gestión del conocimiento, recurso humano, regulaciones, etc. Ver figura 3 [9]. Esta diversidad de actividades y campos de acción técnica y científica explica la existencia de comunidades de materiales que interactúan con la industria, la academia y el consumidor. Algunos ejemplos de organizaciones destacadas son Materials Research Society (www.mrs.org/home/), ASM International (www.asminternational.org/), Federation of Materials Societies (www.materialsocieties.org/), Materials Australia (www. materialsaustralia.com.au/) y Materials UK (www.matuk.co.uk/index.htm) en Gran Bretaña e International Association of Advanced Materials (www.iaamonline.org/), entre otras.

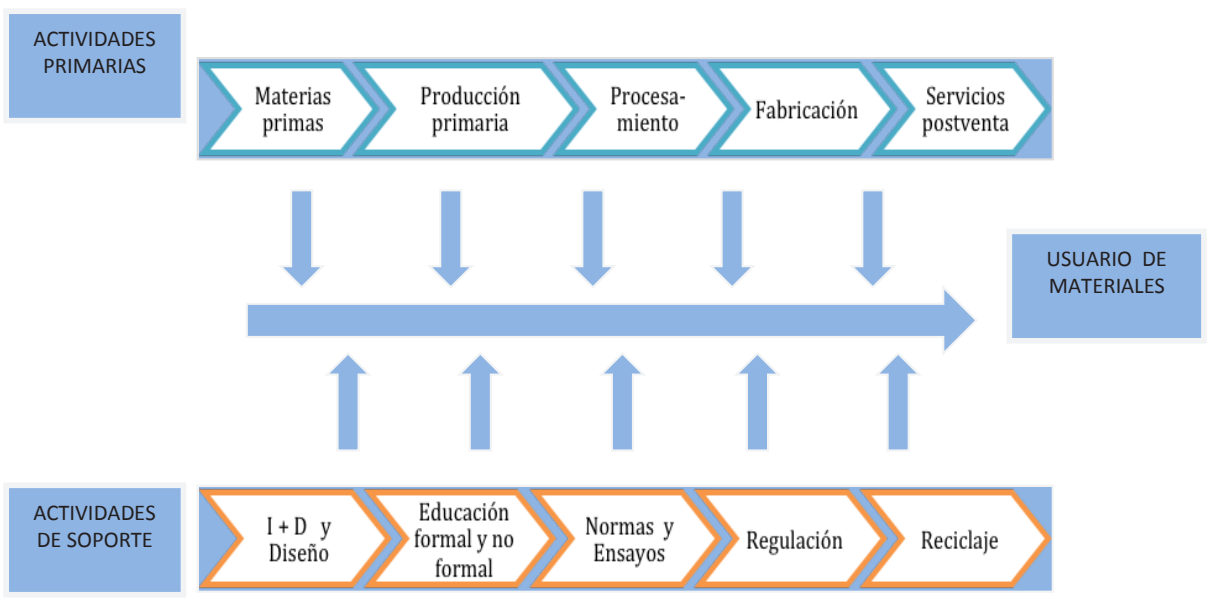

Figura 3. Actividades desarrolladas por la Comunidad de materiales.

Adaptación del autor con base en "A Strategy for Materials" [33] 
Un sistema complejo e interdisciplinario requiere la participación de todos los actores vinculados a la comunidad para facilitar la gestión del conocimiento, en todos los niveles de formación: científicos, ingenieros y técnicos que laboran en la industria, en universidades y en laboratorios de investigación y desarrollo.

\section{FORMACIÓN PROFESIONAL: ÁREAS DE DESEMPEÑO}

De acuerdo con lo descrito, la importancia estratégica de los materiales es consistente con la creciente creación de programas de formación profesional y de posgrado en Ingeniería de Materiales. Por esta razón se hace importante identificar las principales actividades desempeñadas por los ingenieros de materiales en el contexto internacional. El Libro blanco sobre estudios de grado en ingeniería de materiales, de la Agencia Nacional de Evaluación de la Calidad y Acreditación de España [27], distingue cinco grupos de actividades relacionadas con el perfil profesional, ilustrados en la figura 4.
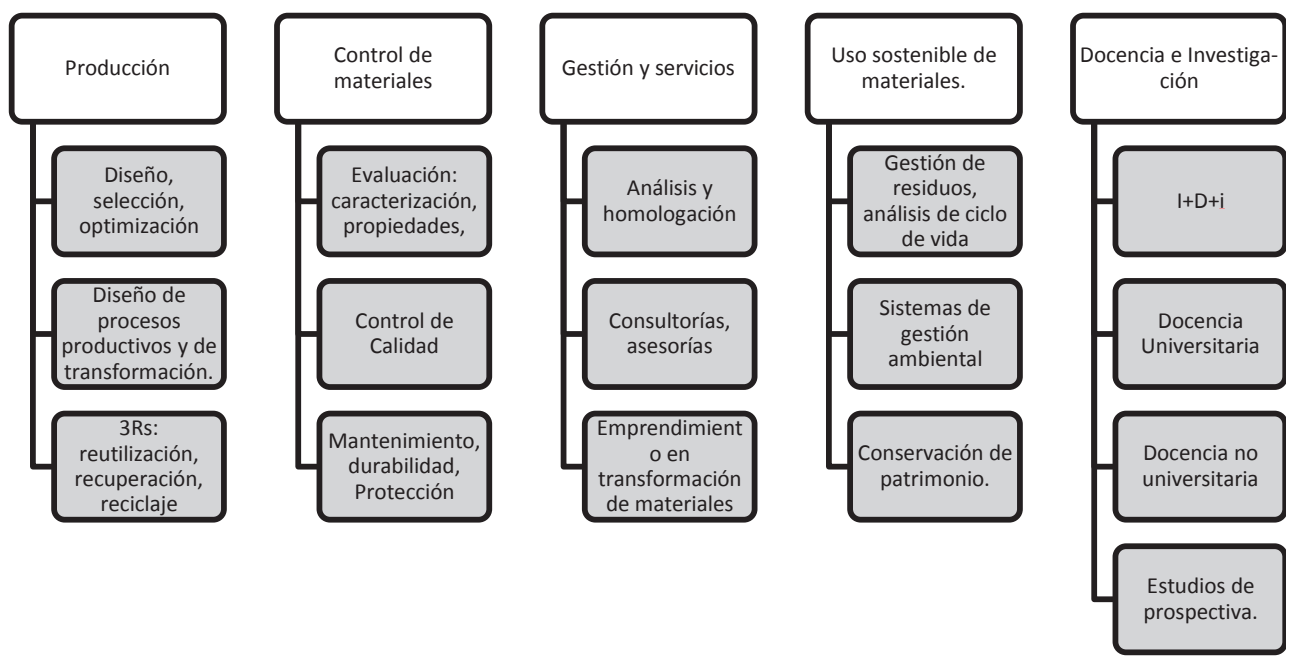

Figura 4. Campos de acción de la ciencia e ingeniería de materiales.

Adaptación del autor de textos libro blanco de la ingeniería de materiales [27]

G1. Producción de materiales: Se relaciona principalmente con el procesamiento en todo el ciclo de vida del material. Desde el beneficio del mineral hasta la obtención de semielaborados: fundición, forja, laminado, estirado, en el caso de la metalurgia; la producción de cerámica tradicional y el desarrollo de nuevos materiales como fibra óptica, revestimientos de barrera térmica y antifricción, hormigones especiales, materiales para disco de freno, aislantes eléctricos, filtros; en procesos productivos de polímeros (inyección, extrusión, etc.), en la evaluación de propiedades y en el desarrollo de nuevos tipos de plásticos o productos con esta materia prima. 
Los materiales compuestos de matriz polimérica y metálica son parte importante de los materiales diseñados (avanzados) que permiten propiedades especiales para el uso en la industria automovilística, aeronáutica, naval, etc. Se debe destacar como áreas emergentes los biomateriales, la biotecnología y la nanotecnología, donde la ingeniería de materiales puede desempeñar un papel importante con la visión sistémica ya descrita, siempre con base en la relación estructura-procesos-propiedades-desempeño.

G2. Control de materiales: Este es un campo de acción de gran impacto en la calidad final de los materiales y productos, clave para enfrentar las exigencias de los mercados globalizados. La caracterización química y física; la evaluación de las propiedades mecánicas, térmicas, magnéticas, etc.; la proyección de la durabilidad y la protección de los materiales frente a agentes del ambiente, entre otras son las actividades más relevantes.

G3. Gestión y servicios relacionados con los materiales: Este renglón de las actividades se puede describir como la gestión de conocimiento y tecnología en el área, desde el sector privado. Es un componente importante de la ocupación en ingeniería en países desarrollados, donde las consultorías y asesorías, el análisis y la homologación de materiales y el emprendimiento son generadores de empleo de buena calidad.

G4. Medio ambiente: uso sostenible de los materiales: Área poco explorada en nuestro medio, pero, frente al daño ambiental generado por el acelerado aumento del consumo de materiales, en un futuro no muy lejano será considerada como de alto impacto económico y social. La gestión de residuos en los sistemas productivos, con el objetivo de aumentar la vida útil, será apreciada desde el punto de vista económico y ambiental, y motivará la especialización de profesionales en esta área.

G5. Investigación y docencia. Los países que han fortalecido el conocimiento científico en el área de materiales han entrado a participar del mercado de producto de alto valor agregado en diversas ramas de la tecnología, con beneficios para sus economías, mejor empleo y mayor capacidad tecnológica. La disminución de la importancia estratégica de las industrias básicas de procesamiento de materiales desde la década del 80 generó grandes cambios en la investigación y en la formación en ingeniería de materiales. A partir de la década del 90 se inició una presión para incluir una gama amplia de materiales en el currículo, la inclusión de una mirada interdisciplinaria y la generación de capacidades de investigación en la formación de los egresados.

\section{EL PANORAMA COLOMBIANO}

El país que desee competir en el mundo global, con alto valor agregado en sus productos, debe realizar desarrollos tecnológicos cada vez más eficientes y competitivos; esto implica construir capacidad para absorber tecnologías foráneas y producir las propias, 
es decir, de innovar en productos, procesos, servicios y en estrategias comerciales, organizativas y financieras [33]. El papel estratégico de los materiales en el diseño de nuevos productos y maquinaria, con calidad y costos competitivos en el mercado global demanda la transferencia de conocimientos entre los sectores académico y productivo.

En Colombia, el área de tecnología relacionada con la transformación de materiales no está incluida en los programas nacionales definidos por Colciencias (http://www. colciencias.gov.co/); las actividades de investigación y desarrollo o propuestas investigativas de universidades y sector productivo quedan inmersas o subyacen en áreas como minería y energía, en biotecnología, en desarrollo industrial, ciencias básicas, entre otros. Este hecho dificulta el fortalecimiento de la comunidad de materiales y la identificación de la ciencia, la ingeniería y la tecnología de materiales como un área estratégica de país, como lo han definido naciones de Europa, Asia y América.

En las últimas décadas han surgido varios programas de posgrado (tabla 2) y grupos de investigación en ciencia e ingeniería de materiales, adscritos tanto a facultades de ciencias (física, química y biología) como a facultades de ingeniería (materiales, química, metalúrgica); a junio de 2014 se encontraban reconocidos por Colciencias 31 grupos de investigación en el área de conocimiento de materiales [34]. Este panorama contrasta con el reducido número de programas de pregrado en el área existente en el país en ingeniería de materiales. Este diagnóstico plantea que la formación de alto nivel, maestría y doctorado, ya es reconocida en nuestro medio y se encuentra en proceso de consolidación, lo que le da valor estratégico a esta área del conocimiento.

Tabla 2. Programas académicos de pregrado, maestría y doctorado en el área de materiales ofrecidos en Colombia, activos según el sistema de información de SNIES del Ministerio de Educación Nacional

\begin{tabular}{|l|c|l|}
\hline \multicolumn{1}{|c|}{ Programas } & Número & \multicolumn{1}{c|}{ Universidades - instituciones } \\
\hline Ingeniería de materiales & 6 & $\begin{array}{l}\text { Antonio Nariño, U. de Antioquia, Autónoma del Caribe, U. del Valle, } \\
\text { San Buenaventura, Universidad de Medellín (en proceso de apertura). }\end{array}$ \\
\hline Especializaciones & 2 & EAFIT, ITM (Instituto Metropolitano Medellín) \\
\hline Maestrías & 8 & $\begin{array}{l}\text { U. de Antioquia, U. Nacional (2), U. Industrial de Santander, U. } \\
\text { Pedagógica y Tecnológica, U. Francisco de Paula Santander, U. del } \\
\text { Valle, U. del Quindío, EAFIT. }\end{array}$ \\
\hline Doctorados & 7 & $\begin{array}{l}\text { U. de Antioquia, U. Pedagógica y Tecnológica, Industrial de Santan- } \\
\text { der, Universidad Nacional (2), U. del Valle, EAFIT. }\end{array}$ \\
\hline
\end{tabular}

Fuente: elaboración propia

Revista Ingenierías Universidad de Medellín 
La labor debe continuar con la formación de profesionales, con las competencias necesarias para diseñar nuevos materiales y optimizar el desempeño de los tradicionales; mejorar la eficiencia en el procesamiento de materiales (menos consumo de materia prima y energía), apoyar las tareas de investigación y desarrollo que emprendan de manera conjunta universidades y empresas. La ingeniería de materiales es un área con un cuerpo de conocimientos consolidado y caracterizada por su interdisciplinaridad, por lo cual su abordaje desde las profesiones tradicionales de la ciencia y la ingeniería arrojará soluciones parciales.

Los retos actuales que enfrentan la ciencia y la ingeniería se caracterizan por su alto grado de complejidad, por lo que requieren tratamiento desde visiones múltiples. La energía, los materiales, el medio ambiente, la biomédica, la biomecánica y la mecatrónica son ejemplos de profesiones emergentes en el campo de la ingeniería que responden a problemáticas tecnológicas de la sociedad [35], a través de la combinación de los saberes y habilidades originados en diferentes disciplinas como la biología, la física, la química en el marco metodológico de la solución de problemas, propio de la ingeniería [36].

\section{REFERENCIAS}

[1] W. D. Callister y D. G. Rethwisch, Materials Science and Engineering: An Introduction, 9th ed., John Wiley \& Sons, Inc, 2013, p. 984.

[2] F. H. A. Janszen y M. P. F. Vloemans, «Innovation and the materials revolution,» Technovation, vol. 17, n. ${ }^{\circ} 10$, p. 549-556, 1997.

[3] C. L. Magee, «Towards quantification of the Role of Materials Innovation in overall Technological Development,» Journal Complexity, vol. 18, n. ${ }^{o}$ 1, pp. 10-25, 2012.

[4] R. Connolly, M. C. Conte, O. Harasic, H. Herrera, G. Redington y D. Vilariño, «Ciencia, tecnología, ingeniería e inovación para el desarrollo: una visión para las Américas en el siglo XXI,» Organization of American States. Office of Education, Science and Technology, Washington, D. C, 2005.

[5] Committee on Condensed-Matter and Materials Physics, Solid State Sciences Committee, Board on Physics and Astronomy, National Research Council, The Physics of Materials:How Science Improves Our Lives, Washintong, DC: National Academic Press, 1997, p. 35.

[6] Unesco, Science for the Twenty-first Century A New Commiyment, A. M. Cetto, Ed., Londodn: A Banson production, 2000, p. 494.

[7] Acatech-Deutsche Akademie der Technikwissenschaften, «Materials Science and Engineering in Germany: Recommendations on Image Building, Teaching and Research,» Fraunhofer IRB Verlag, Munich, 2008.

[8] Committee on Materials Science and Engineering, Solid State Sciences Committee, Commission on Physical Sciences, Mathematics, and Resources, Commission on Engineering 
and Technical Systems, National Research Council, Materials Science and Engineering for the 1990s, Maintaining Competitiveness in the Age of Materials, Washintong D.C: National Academic Press, 1989, p. 281.

[9] Department of Trade and Industry, « Materials Innovation and Growth Team.,» A Strategy for Materials: p. 48, 8 March 2006.

[10] F.-M. Lee, «Materials Science and Engineering Education in Taiwan: a Core Course and a Cornerstone,» Global J. of Engng. Educ., vol. 6, n. ${ }^{\circ}$ 1, pp. 53-58, 2002.

[11] M. Fischer-Kowalsk, A. Alpen-Adria, M. Swilling, E. U. von Weizsäcker, Y. Ren, Y. Moriguchi, W. Crane, F. Krausmann, N. Eisenmenger, Stefan, G. P. Hennicke, R. Kemp, P. R. Lankao y Siriban, UNEP (2011) Decoupling Natural Resource use and Environmental Impacts from Economic Growth, Paris: United Nations Environment Programme, 2011, p. 152.

[12] R. W. Kates, «Our Common Journey: A Transition Toward Sustainability,» National Academy Press, Washignton Dc, 1999.

[13] E. Hertwich, E. van der Voet, S. Suh, A. H. M. Tukker, P. Kazmierczyk, M. Lenzen, J. McNeely y Y. Moriguchi, UNEP (2010) Assessing the Environmental Impacts of Consumption and Production: Priority Products and Materials, A Report of the Working Group on the Environmental Impacts of Products and Materials to the International Panel for Sustainable Resource Manage, Paris: United Nations Environment Programme, 2010, p. 34.

[14] J. Fiksel, «OECD Environment Directorate: 2010 OECD GLOBAL FORUM ON ENVIRONMENT Focusing on SUSTAINABLE MATERIALS MANAGEMENT,» OECD Environment Directorate, OECD, 2010, Mechelen, 2010.

[15] A. Adriaanse, S. Bringezu, A. Hammond, Y. Moriguchi, E. Rodemburg, D. Rogich y H. Schutz, Resource Flows: The Material Basism of Industrial Economies, Tsukuba: World Resources Institute, 1997, p. 64.

[16] M. Fischer-Kowalski, M. Swilling, E. von Weizsäcker, Y. Ren, Y. Moriguchi, W. Crane, F. Krausmann, N. Eisenmenger, S. Giljum, P. Hennicke, P. Romero Lankao, A. Siriban Manalang y Sewerin, UNEP (2011) Decoupling natural resource use and environmental impacts from economic growth, A Report of the Working Group on Decoupling to the International Resource Panel., Paris: United Nations Environment Programme, 2011, p. 83.

[17] H. Nezhad, «WORLD ENERGY SCENARIOS TO 2050: Issues and Options,» Avalaible on the author, [En línea]. Available: : www.nezhadpmd.com

[18] B. Hayman, J. Wedel-Heinen y B. P, «Materials Challenges in Present and Future Wind Energy,» MRS Bulletin, vol. 33, n. ${ }^{\circ}$ 04, pp. 343-353, 2008.

[19] H. Hana, Y. Jeona, S. Limb, W. Kimc y K. Chend, «New developments in illumination, heating and cooling technologies for energy-efficient buildings,» Energy, vol. 35, nº 6, pp. 2647-2653, 2010.

[20] J. W. van der Wiel, «Future of Automotive Design \& Materials Trends and Developments in Design and Materials,» Automotive Technology Center-Acermr.eu, 2011.

[21] L. R. Sproull, «The Early History of The Materials Research Laboratorios» Ann. Rev. Mater. Sci., vol. 17, pp. 1-12, 1987. 
[22] P. L. Hartman, « Cornell's Materials Science Center, The Early Years. Copyright 2007 by the Cornell Center for Materials Research,» [En línea]. Available: http://www.ccmr.cornell.edu/ about/Hartman-MSC-History-2007.pdf [Último acceso: 06 2013].

[23] L. H. Schwartz, «Materials Research Laboratories: Reviewing the First Twenty-Five Years,» de Advancing Materials Research, Washinton D.C, National Research Council, NATIONAL ACADEMY PRESS , 1987, pp. 35-50.

[24] S. F. William, Fundamento de La Ciencia e Ingeniería de Materiales, vol. $3^{\circ}$ ed, Mc Graw Hill, 1998, p. 368.

[25] The Higher Education Academy, and UK Center for Materials Education, « National Subject Profile for higher education programmes in Materials,» The Higher Education Academy, Heslington, 2008.

[26] M. C. Flemings y R. W. Cahn, «Organization and trends in Materials Science and Engineering Education in the US and Europe,» Acta Mater, vol. 48, pp. 371- 383, 2000.

[27] Agencia Nacional de Evaluación de la Calidad y Acreditación de España, «Libro Blanco. ESTUDIOS DE GRADO EN INGENIERÍA DE MATERIALES,» Universidad Politécnica de Madrid, Madrid, 2005.

[28] Ministério da Educação - Sistema e-MEC, «Instituições de Educação Superior e Cursos Cadastrados,» Ministério da Educação, 2014. [En línea]. Available: http://emec.mec.gov.br/ [Último acceso: 302 2014].

[29] CAPES, «Mestrados/Doutorados Reconhecidos,» CAPES, 2006 2014. [En línea]. Available: http://conteudoweb.capes.gov.br/conteudoweb/ProjetoRelacaoCursosServlet?acao=pesquisa

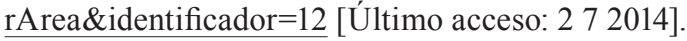

[30] Ministerio de Educación, «Guía de Carreras Universitarias,» Ministerio de Educación- Argentina, 2014. [En línea]. Available: http://ofertasgrado.siu.edu.ar/carreras_de_posgrado.php [Último acceso: 27 2014].

[31] Ministerio de Educación-Chile, «Mifuturo.cl,» Ministerio de educación, [En línea]. Available: http://www.mifuturo.cl/index.php/donde-y-que-estudiar/buscador-de-carreras [Último acceso: 27 2014].

[32] L. A. Dobrzanski, «Significance of materials science for the future development of societies,» Journal of Materials Processing Technology, vol. 175 , p. 133-148, 2006.

[33] F. García-Córdoba, La Investigación tecnológica: investigar, idear e innovar en ingenierías y ciencias sociales, México: Limusa, 2005, p. 452.

[34] Colciencias, «Cienmcia y Tecnología para Todos,» Corporación Ciencia y Tecnología y Sociedad, [En línea]. Available: http://scienti.colciencias.gov.co:8083/ciencia-war/BusquedaGruposXAreaDetalles.do?codAreaConocimiento $=0000000000 \& \operatorname{codRh}=2 \mathrm{E}$ [Último acceso: 27 2014].

[35] Zehev Tadmor, «Redefining Engineering Disciplines for the Twenty-First Century,» The Bridge, vol. 36, n 2, pp. 33-37, Summer 2006. 
[36] R. J. M. Vélez, «Propuesta de creación un programa de pregrado en el área de ciencia, ingeniería y tecnología de materiales. Documento de trabajo,» Universidad Nacional Sede Medellín (http://www.bdigital.unal.edu.co/11680/1/JuanManuelV\%C3\%A9lezRestrepo.pdf), Medellín, 2013. 